\title{
Shock Wave Model for Sputtering Biomolecules Using Massive Cluster Impacts
}

\author{
John F. Mahoney and Julius Perel \\ Phrasor Scientific, Inc., Duarte, California, LSA \\ Terry D. Lee and P. A. Martino \\ Division of Immunology, Beckman Research Institute of the City of Hope, Duarte, California, USA
}

\author{
Peter Williams \\ Department of Chemistry, Arizona State University, Tempe, Arizona, USA
}

A shock wave model is proposed to explain certain features of recently reported spectra obtained by massive cluster impact (MCI) mass spectrometry. It is suggested that clusters that impact glycerol matrices with energies/nucleon in the range $0.01 \mathrm{eV} / \mathrm{u}<\mathrm{E} / \mathrm{N}<1.0$ $\mathrm{eV} / \mathrm{u}$ provide an extremely soft method for sputtering intact biomolecules. Compared to the high energy/nucleon characteristic of atomic or molecular ion primary beams (typically $<50 \mathrm{eV} / \mathrm{u}$ ), massive cluster primary beams possess much lower energies/nucleon, which are insufficient to cause appreciable ionization and radiation damage of matrix material. Moreover, fragmentation products of parent molecular ions are effectively lower. With these benefits, MCI spectra show lower chemical noise background and enhanced signalto-noise ratios. Rankine-Hugoniot analysis of the shock conditions is used to arrive at an estimate of the heat retained in the collision-affected matrix volume after bombardment by a characteristic cluster. For a cluster collision resulting in a $26.8 \mathrm{GPa}$ shock pressure, by analogy with water data, rapid heating of the shocked volume to $100{ }^{\circ} \mathrm{C}$ or more is plausible. In a beam consisting of clusters distributed in size and charge, an estimate is made for the range of cluster sizes over which hyrodynamic shock wave theory applies. $(J$ Am Soc Mass Spectrom 1992, 3, 311-317)

W e have recently reported on a new technology for ion desorption that uses a primary beam of massive, multiply charged clusters to generate secondary ions of peptides in a glycerol matrix [1]. This novel sputtering method has been termed massive cluster impact (MCI) mass spectrometry. Spectra obtained by MCI are characterized by a near absence of fragment ions and greatly reduced background derived from matrix ions. A comparison of the negative ion spectra of a peptide obtained by using $\mathrm{MCI}$ and $\mathrm{Xe}$ fast atom bombardment (FAB) is shown in Figure 1. Two glycerol cluster series account for the majority of the prominent ions in the low mass region of the spectrum. One series is derived from adducts with acetate anions $(\mathrm{m} / \mathrm{z} 59)$ that results, in part, from debris from the glycerol clusters used to bombard the sample. Ammonium acetate is used as the electrolyte in the primary beam solution. The second glycerol series is derived from adducts with

Address reprint requests to John F. Mahoney, Phrasor Scientific, Inc., 1536 Highland Avenue, Duarte, CA 91010 trifluoroacetate (TFA) anions ( $m / z$ 113). The peptide sample exists as the TFA salt. Only a few weak fragment ions are seen, resulting from the loss of small neutral molecules from the deprotonated molecule. In contrast, the matrix ions in the corresponding xenon $\mathrm{FAB}$ spectrum are much more intense relative to the signal for the deprotonated molecule (ions at $100 \%$ relative intensity below $\mathrm{m} / \mathrm{z}$ 300 are saturated). In addition to the ion series described for the MCI spectrum, there is the usual dominant glycerol series derived from the deprotonated glycerol anion, which is very weak in the $\mathrm{MCI}$ spectrum. In the higher mass region of the FAB spectrum, peptide fragment ions are readily assigned. Both the FAB and the MCI spectra show adducts of the intact molecule with TFA.

In this article we present the view that the sputtering of large molecules from a liquid matrix initiated by the hypervelocity impact of massive, multiply charged clusters can be described as a classic macroscopic shock wave phenomenon. Shock wave models $[2,3]$, used to explain nonlinear effects in observed sputter- 

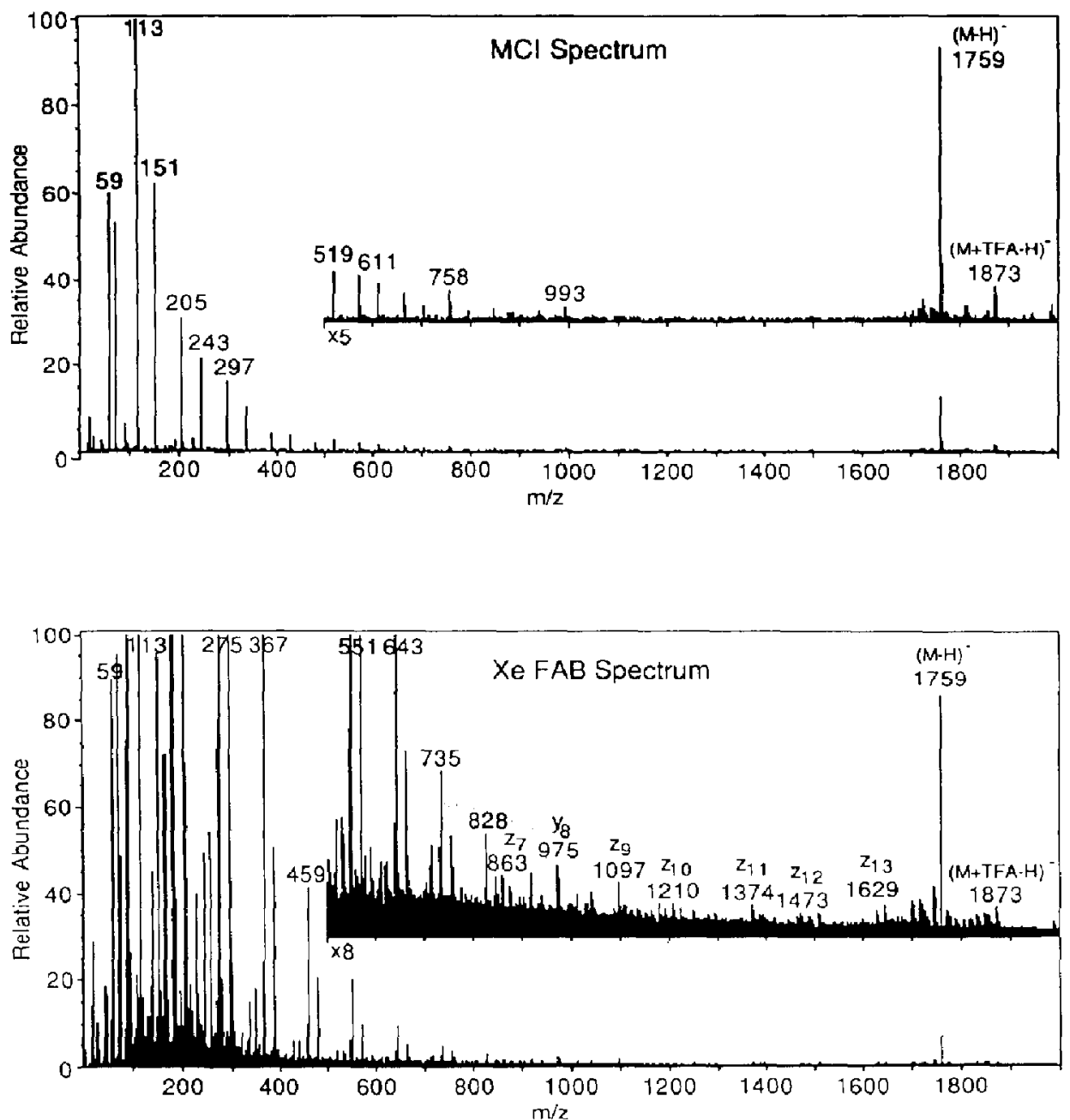

Figure 1. Comparison of the negative-ion spectrum of the peptide Preangiotensin (MW 1760, sequence DRVYIHPFHLVTHN) obtained by using a massive cluster ion beam (upper) and Xe FAB (lower). The peptide sample ( 2 nmol) was dissolved in a glycerol matrix and analyzed by using a JEOL HX100HF magnetic sector mass spectrometer (JEOL, USA) operating at 5-kV acceleration potential and a nominal resolving power of 500. A 1.5-M solution in glycerol: water (30:70) was used to generate the primary ion beam by $\mathrm{EH}$ emission. Initial beam energy was $13 \mathrm{keV}$. A Xe atom beam with 6-keV translational energy was used for the FAB spertrum.

ing yields, have been proposed for primary beams consisting of $80 \mathrm{MeV}$ fission fragments [4], $14 \mathrm{MeV}$ neutrons [5], keV atomic ions [6], and million electronvolts atomic and diatomic ions. For shock waves to form in targets bombarded by atomic or molecular ions, a mechanism must exist that ultimately provides a collective motion of target material. One such mechanism [4] proposes an ion explosion model whereby coulombic repulsion in the initial ionization track is transformed into kinetic energy of the ions. Collision of the energetic ions with the surrounding matter provides the compression necessary to propagate a shock wave.
The macroscopic shock wave model proposed herein for sputtering by primary cluster beams appears valid in view of the massiveness $\left(10^{6}-10^{7} u\right)$ of the clusters that impact with velocities exceeding the velocity of sound in the target material. Moreover, sputtering precipitated by elastic nuclear collisions or electronic processes seems improbable due to the inherently small cluster energy/nucleon (less than 1.0 $\mathrm{eV} / \mathbf{u}$ ). Upon cluster impact, the molecules comprising the matrix material are set in coherent motion by a strong compressional wave. Instantaneously, a second shock wave is propagated through the cluster causing its disintegration shortly after impact. 


\section{Electrohydrodynamically Generated Clusters}

Clusters treated in this study are formed by the electrohydrodynamic (EH) atomization of glycerol solutions in vacuum. Details of cluster source construction and molecular ion spectra generated by MCIs are discussed elsewhere [1]. Far from being monodisperse, charged particle beams generated by the EH method consist of cluster ensembles distributed both in size and charge state. Generally, the heavy cluster continuum is accompanied by a residual low mass molecular ion beam component. Typically, the small ion component in the bcams represents about $10 \%$ of the total cluster beam current. To alleviate the analytical complexities involved with distributions, a mean cluster size representative of the distribution is chosen for deriving the physical parameters subsequently used in shock wave analysis. For this analysis the primary parameter of interest is the impact velocity, $u_{0}$, given by

$$
v_{0}=(2(q / m) V)^{\frac{1}{2}}
$$

where $\langle q / m\rangle$ is the mean cluster charge-to-mass ratio and $V$ is the net acceleration potential. In general, $\langle q / m\rangle$ is determined from the experimentally measured values of cluster source emission current $i$ and flowrate, $d m / d t$, of the atomized solution according to

$$
(q / m)=i(d m / d t)^{-1}
$$

For $i=10 \mu \mathrm{A}$ and $d m / d t \approx 10^{-8} \mathrm{~g} / \mathrm{s}$ (typical experimental values), $(q / m) \approx 10^{3} \mathrm{C} / \mathrm{kg}$. Substitution of this value for $\langle q / m\rangle$ into eq 1 and taking $V=18 \mathrm{kV}$, the impact velocity $v_{0}$ becomes $6 \mathrm{~km} / \mathrm{s}$, which is $\approx 3$ times the velocity of sound in a glycerol matrix (1.9 $\mathrm{km} / \mathrm{s}$ ).

\section{Shock Wave Model}

With reference to Figure 2, we will consider the onedimensional case of a normal collision involving a glycerol cluster that impacts a glycerol target with velocity $v_{0}$. Upon impact a compressive shock wave of velocity $U$ is propagated into both the cluster and the glycerol matrix. Applying conservation relations for momentum, mass, and energy across the shock fronts results in the following relationships:

$$
\begin{gathered}
p-P_{0}=\rho_{0} u U \\
V / V_{0}=(U-u) / U \\
E-E_{0}=\frac{1}{2}\left(P+P_{0}\right)\left(V_{0}-V\right)
\end{gathered}
$$

called the Rankine-Huganiot equations. The derivation of these relationships can be found in a number of texts [7-9]. Here $P_{0}, V_{0}, E_{0}$, and $\rho_{0}$ are the pres-

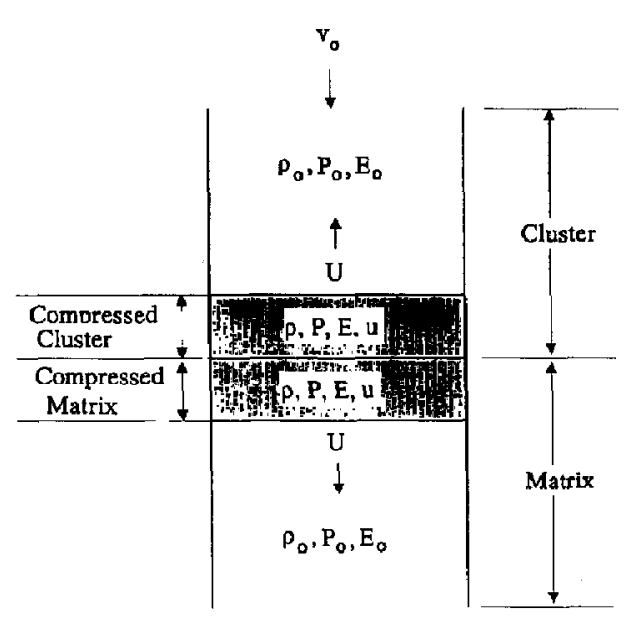

Figure 2. Shock wave propagation in cluster and matrix.

sure, specific volume, specific energy, and density of the undisturbed media in front of the shock wave. The corresponding quantities relating to compressed material behind the shock wave are $P, V, E$, and $\rho$ with $u$ denoting the particle velocity behind the shock front.

To estimate how the initial impact energy is partitioned between the bombarding cluster and matrix leading eventually to a determination of the amount of irreversible heating of the matrix, it is necessary to graphically construct a $P, V$ equation of state under shock conditions known as the Hugoniot curve. With the aid of Figure 3, a brief description of the Hugoniot curve is included here because of its importance to the ensuing analysis. After impact with the cluster, the matrix material is compressed from an initial $P_{0}, V_{0}$ state $\mathrm{A}\left(P=0, V / V_{0}=1\right)$ to the funal $P, V$ state $\mathrm{B}$, where $P$ is taken as the impact or shock pressure. The intermediate points on the Hugoniot curve $A B$

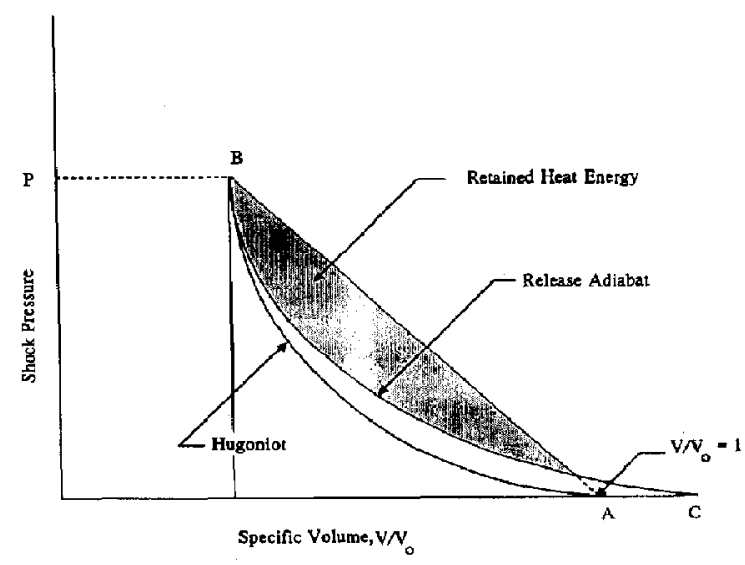

Figure 3. Shock pressure versus specific volume. 
represent the locus of $P, V$ states reached by discontinuous pressure jumps across the shock front. The matrix returns to ambient conditions along the release or decompression isentrope $\mathrm{BC}$ by rarefaction waves. Note that the area $A$ under the dashed triangle is $\frac{1}{2} P\left(\mathbf{1}-V / V_{0}\right)$. After dividing by $\rho_{0}$, this area represents the change in specific internal energy per unit mass of the shock compressed matrix, that is, $\Delta E / m$ $=A / \rho_{0}$. This value is equivalent to the Hugoniot energy relationship when $P \gg P_{0}$ because, from eq 3.

$$
\frac{\Delta E}{m}=\frac{P}{2 \rho_{0}}\left(1-\frac{V}{V_{0}}\right)
$$

As the area under the release isentrope represents the PV work done by adiabatic expansion of the matrix material to ambient conditions, the difference between this area and the dashed triangle is proportional to residual heat left in the matrix after decompression. Subsequent analysis will attempt to roughly estimate the spccific heat energy per unit mass retained by the matrix after impact with a characteristic cluster having an impact velocity $v_{0}=6 \mathrm{~km} / \mathrm{s}$. This value can then be compared to the specific cnergy required to vaporize glycerol.

Fortunately, experimental shock data for glycerol exists for which a glycerol I lugoniot curve can be constructed [10]. The procedure to do so is outlined as follows. By using the values for shock velocity $U$ and particle velocity $u$ tabulated in Table 1 , the shock pressure $P$ and the relative volume $V / V_{0}$ can be computed by using the Rankine-Hugoniot eqs 3 and 4. Figure 4 illustrates the approximate linear dependence of shock velocity on particle velocity. The glycerol Hugoniot curve showing the relative volume $V / V_{0}$ as a function of shock pressure is graphically depicted in Figure 5.

Table 1. Glycerol shock data [10]

\begin{tabular}{ccccc}
\hline $\begin{array}{c}\rho \\
\left(\mathrm{g} / \mathrm{cm}^{3}\right)\end{array}$ & $\begin{array}{c}U \\
(\mathrm{~km} / \mathrm{s})\end{array}$ & $\begin{array}{c}u \\
(\mathrm{~km} / \mathrm{s})\end{array}$ & $\begin{array}{c}P \\
(\mathrm{GPa})\end{array}$ & $V / V_{\mathrm{O}}$ \\
\hline \hline 1.248 & 1.900 & 0.000 & 0.000 & 1.000 \\
1.384 & 2.650 & .260 & .860 & .902 \\
1.446 & 3.000 & .410 & 1.535 & .863 \\
1.553 & 3.460 & .680 & 2.936 & .803 \\
1.580 & 3.620 & .760 & 3.433 & .790 \\
1.642 & 3.920 & .940 & 4.599 & .760 \\
1.766 & 4.525 & 1.278 & 7.281 & .718 \\
1.796 & 4.620 & 1.410 & 8.130 & .695 \\
1.861 & 5.310 & 1.750 & 11.597 & .670 \\
1.940 & 5.770 & 2.020 & 14.697 & .650 \\
1.971 & 5.997 & 2.184 & 16.411 & .636 \\
2.049 & 6.650 & 2.600 & 21.578 & .609 \\
2.083 & 6.760 & 2.710 & 22.853 & .599 \\
2.187 & 7.220 & $\mathbf{3 . 1 0 0}$ & 27.933 & .571 \\
2.194 & 7.770 & $\mathbf{3 . 3 5 0}$ & 32.486 & .569 \\
2.293 & 8.800 & 4.010 & 44.039 & .544 \\
2.473 & 9.530 & 4.720 & 56.137 & .505 \\
\hline
\end{tabular}

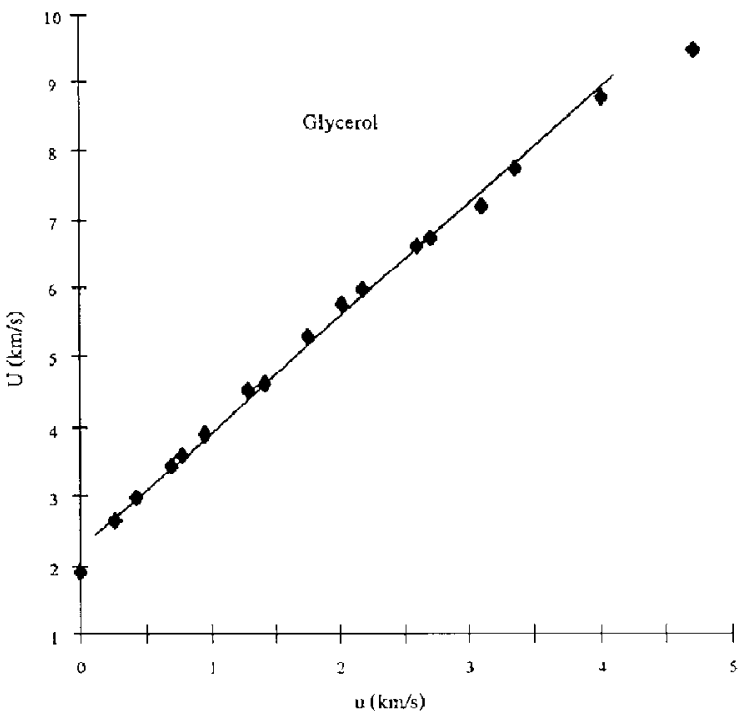

Figure 4. Shock velocity in glycerol as a function of particle velocity

\section{Estimate of Heat Energy Retained by Matrix}

To estimate the percentage of the cluster impact energy imparted to the matrix in the form of heat energy, the shock pressure associated with a characteristic cluster impacting with a velocity $n_{0}=6 \mathrm{~km} / \mathrm{s}$ must be determined. It can be shown that, for a symmetrical impact involving similar materials, the impact velocity is equal to the sum of the shocked particle velocities in the glycerol cluster and matrix target, or $v_{0}=2 u$ [11]. Thus, $u=3 \mathrm{~km} / \mathrm{s}$ which, from inspection of Figure 4 , corresponds to a shock velocity of 7.1 $\mathrm{km} / \mathrm{s}$. Substitution of these values into the Rankine-

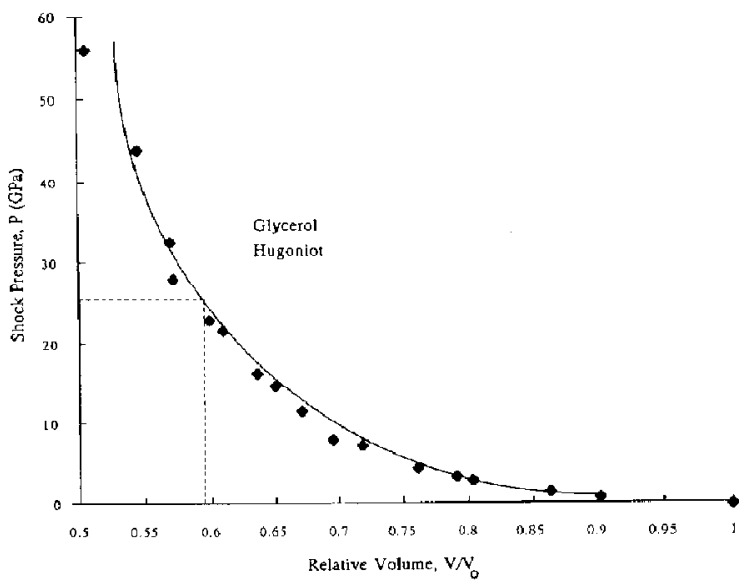

Figure 5. Hugoniot curve for glycerol. 
Hugoniot relation $P=\rho_{0} u U$ (using $\rho=1: 26 \times 10^{3}$ $\mathrm{kg} / \mathrm{m}^{3}$ ) yields $26.8 \mathrm{GPa}$ for the shock pressure indicated by the horizontal dashed line in Figure 5.

To circumvent complexities involved with calculating the release adiabat ${ }_{r}$ the heat energy stored in the matrix after decompression can be estimated by taking the Hugoniot curve as an approximation for the decompression adiabat, an approach suggested by Gault and Heitowit [11]. As pointed out by these authors, the overestimation of the heat energy by this method is only about a factor of 2. By using this approximation, the specific heat energy, $\Delta H / m_{r}$ becomes

$$
\begin{aligned}
\Delta H / m= & \left(P / 2 \boldsymbol{\rho}_{0}\right)\left(1-V / V_{0}\right) \\
& -(\text { Area under Hugoniot }) / \boldsymbol{\rho}_{0}
\end{aligned}
$$

The area under the Hugoniot was calculated by first fitting the Hugoniot curve (Figure 5) with the analytical expression $y(x)=a+b \exp (-k x)$. The constants $a, b, k$ have the following values: $a=-0.507, b=$ $8.18 \times 10^{3}$, and $k=9.69$. Integrating this expression and evaluating the result between the limits $V / V_{0}=$ $0.588(P=26.8 \mathrm{GPa})$ and $V / V_{0}=1(P=0)$ yields an area $=2.56 \times 10^{9} \mathrm{GPa}$. Finally, the specific heat energy computed from eq 7 is $2350 \mathrm{~kJ} / \mathrm{kg}$. Taking half this value, to be more realistic in view of the above approximation, $\Delta H / m=1175 \mathrm{~kJ} / \mathrm{kg}$, which compares favorably with the heat of vaporization for glycerol $\approx$ $1000 \mathrm{~kJ} / \mathrm{kg}$. Thus, after decompression, the affected volume of glycerol may be left as a heated liquid and in a partially vaporized state.

The total specific energy available in a cluster impact is $v_{0}^{2} / 2$, which for a cluster with impact velocity $v_{0}=6 \mathrm{~km} / \mathrm{s}$ corresponds to $1.8 \times 10^{7} \mathrm{~J} / \mathrm{kg}$. By using the figure arrived at above for $\Delta H / m$, the percentage of the total specific energy imparted to the matrix in the form of retained heat is estimated at $6.5 \%$. Shock temperatures for water of about $1500^{\circ} \mathrm{C}$ have been reported when shocked to pressures near $26.8 \mathrm{GPa}$ [12].

\section{Limits on Cluster Sizes for Shock Wave Formation}

As might be expected, for a given acceleration vollage a limited range of cluster sizes exists below and above which hydrodynamic shock waves may not form in the impacted matrix. The minimum cluster size below which primary shock waves will not form is determined by the interaction time involved in the clustermatrix collision. If this time is of the order or smaller than molecular vibration times $\left(\approx 10^{-12} \mathrm{~s}\right)$, macroscopic shock wave processes governed by continuum mechanics may not apply. Clusters with interaction times approaching molecular vibration periods can be estimated in the following manner. By setting the interaction time $t$ equal to the time required for a cluster to disintegrate after impact, $t=2 D_{\min } / U$ where $D_{\min }$ is the cluster diameter and $U$ is the shock velocity. A factor of 2 appears in the expression for $t$ because once the compressional shock wave travels the cluster diameter, it is reflected from the free surface of the cluster as a rarefaction wave with velocity $U_{r}$, where $U_{r} / U \approx 1.0$ [13]. The cluster is assumed to disintegrate after passage of the rarefaction wave. The requirement that the interaction time exceed molecular vibration times $t$ is expressed as $\left(2 D_{\min } / U\right)>\tau$. Taking the impact velocity $v_{0} \approx U$, the minimum cluster size becomes $D_{\min }>\left(v_{0} \tau\right) / 2$. The impact velocity in this last expression must be replaced by its functional dependence on cluster size by using eq 1 to subsequently arrive at a final expression for $D_{\min }$.

After substituting for the most probable value of $\langle q / m\rangle$ in eq 1 by using [14]

$$
\langle q / m\rangle=3\left(\epsilon_{0} \gamma\right)^{1 / 2} / \rho_{0} r^{3 / 2}
$$

where $\epsilon_{0}$ is the permittivity of free space, $\gamma$ and $\rho_{0}$ are the cluster surface tension and density, and $r$ is the cluster radius, the minimum cluster diameter becomes

$$
D_{\min }=1.51\left[\left(\epsilon_{0} \gamma\right)^{1 / 2} V / \rho_{0}\right]{ }^{.286}{ }^{.571}
$$

For glycerol clusters accelerated through a potential $V=10 \mathrm{kV}$ and setting $r=10^{-12} \mathrm{~s}$ in eq 9, clusters with diameters greater than $\approx 6.8 \times 10^{-4} \mathrm{~m}(.0068 \mu)$ interact with the matrix with collision times greater than $10^{-12} \mathrm{~s}$.

The maximum cluster size above which shock waves are extremely weak or will not form is determined by clusters that impact matrices with velocities equal to or below the speed of sound in glycerol $(c=1.9 \mathrm{~km} / \mathrm{s})$. An expression for estimating the maximum cluster size is found by setting the impact velocity $v_{0}=c$ in eq 1 and solving for $\langle q / m\rangle$. Substitution of the resultant expression obtained for $\langle q / m\rangle$ into eq 8 and solving for $D_{\max }$ gives the following

$$
D_{\max }=2\left[6\left(\epsilon_{0} \gamma\right)^{1 / 2} V / \rho_{0} c^{2}\right]^{2 / 3} .
$$

Letting $V=10 \mathrm{kV}, \gamma=0.063 \mathrm{~N} / \mathrm{m}, \rho_{0}=1.26 \times 10^{3}$ $\mathrm{kg} / \mathrm{m}^{3}$, we find that clusters with $D_{\max }>9.18 \times 10^{-8}$ $\mathrm{m}$ are less likely to form shock waves after impacting the matrix. The ratio $D_{\max } / D_{\min }=13.5$ indicating that, for a beam containing a wide distribution of clusters, cluster sizes with over an order of magnitude range within the distribution can cause shock wave formation.

\section{Impact Regimes for Cluster Distribution}

The predicted range of cluster sizes that can generate shock waves in matrices for a given acceleration voltage is shown in Figure 6 as a function of energy/ nucleon (electronvolt per unit). Additionally, Figure 6 


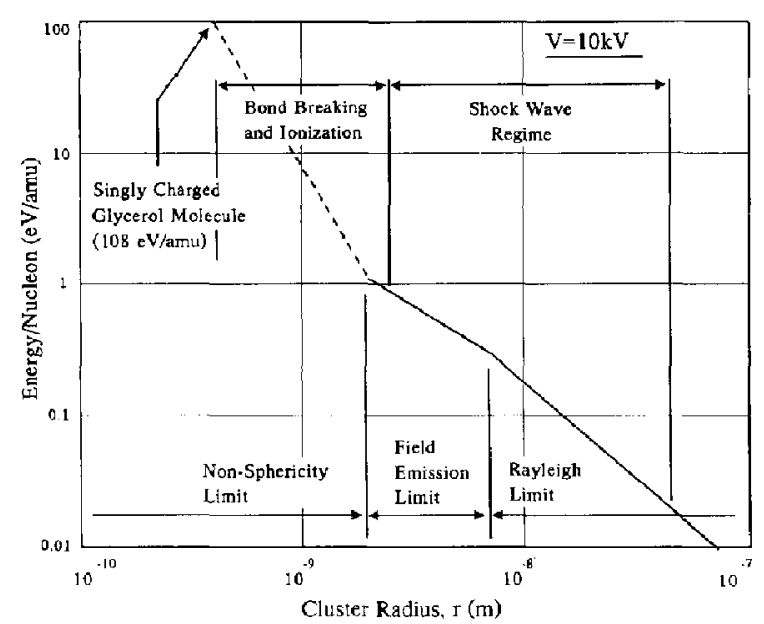

Figure 6. Energy/nucleon dependence on glycerol cluster size (Accel. voltage $=10 \mathrm{kV}$ ).

illustrates the impact regimes predicted for an entire cluster distribution (including the small mulecular ion component) and the type of sputtering interaction expected when clusters bombard a matrix. In calculating the energy/nucleon for the cluster distribution, clusters are assumed charged to their maximum limits. For larger clusters the maximum charge is governed by the modified Rayleigh criterion (eq 8), and for smaller clusters the maximum charge $q$ allowable is determined from the field emission limit [15] given by

$$
q=4 \pi e_{0} r^{2} E_{\text {ion }}
$$

where $E_{\mathrm{ion}}$ is the onset electric field necessary to field evaporate charge from clusters. The transition region separating the Rayleigh limit from the field emission limit is found by equating eq 11 to eq 8 and solving for the cluster radius $r$.

Clearly, departure of smaller clusters from sphericity invalidates the use of eq 11, which assumes spherical clusters for calculation of cluster charge. At the extreme, a singly charged glycerol molecule (assumed to have a diameter of 4-5 $\AA$ ) has an energy/nucleon of $108 \mathrm{eV} / \mathrm{u}$. This value is extrapolated (dashed line in Figure 6) to an assumed value of cluster radius where clusters depart from sphericity.

The number of nucleons $N$ in a spherical cluster of radius $r$ in either the Rayleigh or field emission regime is given by

$$
N=4 \pi \rho_{0} r^{3} / 3 m_{p}
$$

where $m_{p}$ is the proton rest mass. Within the Rayleigh limit, the cluster energy $E_{R}$ expressed in electronvolts is

$$
E_{R}=4 \pi r^{3 / 2}\left(\epsilon_{0} \gamma\right)^{1 / 2}(V / e)
$$

where $V$ is the potential through which clusters have been accelerated and $e$ is the electronic charge. The energy/nucleon as a function of cluster size in the Rayleigh limit regime is found by dividing eq 13 by eq 12, yielding

$$
E_{R} / N=3\left(\epsilon_{0} \gamma\right)^{1 / 2} V m_{p} / e \rho_{0} r^{3 / 2}
$$

In the field emission regime, the cluster energy $E_{F}$ is

$$
E_{F}=4 \pi \epsilon_{0} r^{2} E_{\mathrm{ion}} V / e
$$

resulting in an energy/nucleon of

$$
E_{\mathrm{F}} / N=3 \epsilon_{0} E_{\mathrm{ion}} V m_{p} / e \rho_{0} r
$$

Referring to Figure 6, note that for $V=10 \mathrm{kV}$, the shock wave region for sputtering encompasses cluster sizes over nearly the entire field emission and Rayleigh regimes corresponding to energies/nucleon in the range $.01 \mathrm{eV} / \mathbf{u}<E / N<1.0 \mathrm{eV} / \mathrm{u}$.

\section{Discussion}

Compared to the high energies/nucleon encountered in collisions involving atomic or molecular primary beams (typically greater than $50 \mathrm{eV} / \mathrm{u}$ ), massive clusters impact in the shock wave regime with much lower energies/nucleon. At these low energy/nucleon impacts, ionization and radiation damage of matrix material are expected to be minimal. This in fact appears to be the case as evidenced by recent comparisons of $\mathrm{MCl}$ spectra with atomic ion primary beam spectra [1]. The MCI spectra demonstrated lower chemical noise and higher signal-to-noise ratios. Moreover, fragmentation of parent molecular ions was effectively eliminated. Smaller clusters and low mass molecular ions persisting in cluster beams can, however, produce some intramolecular bond breaking and ionization of matrix material. Future work with $\mathrm{MCl}$ beams will examine ways for filtering out the higher energy/nucleon species expected to result in even cleaner spectra.

Although a shock model is proposed herein to describe the sputtering process for large biomolecules using multiply charged, massive cluster primary beams, it is far from complete. Once an initiating vehicle (i.e., large clusters) is identified that is responsible for propagating shock waves in a matrix, the physical mechanisms governing subsequent material sputtering may closely follow those proposed for other shock induced processes, for example, pulsed laser ablation [16]. Here rapid shock heating is suggested that causes intermolecular bond breaking accompanied by material vaporization and ejection. Ultimately, for any sputtering model to be successful, it should correctly predict secondary ion yields as a 
function of the physical properties of the primary beam.

\section{Acknowledgments}

The authors gratefully acknowledge the financial support of the National Institute of Health for this work (Phase I SBIR grant no. 1 R43 GM45656-01, JFM) (R01 GM40673-03, TDL). We also thank Dr. T. J. Ahrens (California Institute of Technology) for helpful discussions regarding shock wave phenomena. We are also grateful to Dr. B. Kalensher and Dr. E. Parilis for many helpful discussions.

\section{References}

1. Mahoney, J. F.; Perel, J.; Ruatta, S. A.; Martino, P. A.; Husain, S.; Lee, T. D. Rapid Comm. Mass Spectrom. 1991, 5, 441-445.

2. Carter, G. Rad. Eff. Letts. 1979, 43, 193-199.

3. Carter, G. Nucl. Instr. and Meth. 1983, 209/210, 1-11.

4. Hayashiuchi. Y.; Kitazoe, Y.; Sekiya, T.; Yamamura, Y. J. Nucl. Mat. 1977, 71, 181-186.

5. Guinan, M. J. Nucl. Mat. 1974, 53, 171-178.
6. Kitazoe, Y.; Yamamura, Y. Rad, Eff. Letts. 1980, 50, 39-44.

7. Courant, R.; Friedrichs, K. O. Supersonic Flow and Shock Waves; Interscience: New York, 1948.

8. Lindsay, R. B. Mechanical Radiation; McGraw-Hill: New York, 1960.

9. McQueen, R. G.; Marsh, S. P.; Taylor, J. W.; Fritz, J. N.; Carter, J. W. In High-Velocity Impact Phenomena; Kinslow, R., Ed.; Academic: New York, 1970.

10. Marsh, S. P., Ed. LASL Shock Hugoniot Data; University of California: Berkeley, CA, 1970.

11. Gault, D. E.; Heitowit, E. D. In Proceedings of the 6 th Symposium on Hyperolocity Impact: Vol. II, Part 2; Cleveland, OH, Aprtl 30-May 3, 1963; AD 423064.

12. Ahrens, T. J.; O'Keefe, J. D. In Ices In The Solar System; Klinger, J., Ed.; D. Reidel Publishing Co., 1985.

13. Rice, M. H, MçQueen, R. G.; Walsh, J. M. In Solid State Physics: Vol. 6; Seitz, F.; Turnbull, D., Eds.; Academic: New York, 1958.

14. Pfeifer, R. J.; Hendricks, C. D. Phys. Fluids 1967, 10, 2149-2154.

15. Korhn, V. E. I. Appl. Phys. 1974, 47, 1144-1146.

16. Williams, P.; Nelson, R. W. In Methods and Mechanisms for Producing Ions from Large Molecules; Standing, K. G. and Ens, W., Eds.; Plenum: New York, 1991; p 265. 